\title{
Lung transplantation in six patients with idiopathic pulmonary artery hypertension
}

\author{
Hyeonhwa Kim', Dongkwan Kim², Sehoon Choi ${ }^{2}$, Geundong Lee ${ }^{2}$, Dongkyu $\mathrm{Oh}^{3}$, Jaeseung Lee ${ }^{3}$, Sungho Jung ${ }^{2}$, \\ Seungill Park ${ }^{2}$, Sangbum Hong ${ }^{3}$ \\ ${ }^{1}$ Department of Internal Medicine, Asan Medical Center, University of Ulsan College of Medicine, Seoul, Korea
${ }^{2}$ Department of Thoracic and Cardiovascular Surgery, Asan Medical Center, University of Ulsan College of Medicine, Seoul, Korea
${ }^{3}$ Department of Pulmonary and Critical Care Medicine, Asan Medical Center, University of Ulsan College of Medicine, Seoul, Korea
}

Background: Idiopathic pulmonary artery hypertension (IPAH) is an incurable and invariably fatal disease. Lung transplantation is a useful therapeutic option in patients who are unresponsive to medical treatment; however, lung transplantation performed for pulmonary hypertension is associated with significantly high perioperative mortality rates.

Methods: We report case series of six patients who underwent lung transplantation for IPAH between October 2008 and June 2021.

Results: Patients' median age was 28.5 years, and the study included 5 of 6 female (83\%). Pre-transplantation hemodynamic parameters showed mean right atrial pressure of $12.0 \pm 7.1 \mathrm{mmHg}$ and mean pulmonary artery pressure of $62.2 \pm 29.5 \mathrm{mmHg}$. Two of six patients received extracorporeal membrane oxygenation (ECMO) therapy as a bridge to transplantation over 14 and 17 days, and four patients underwent elective transplantation. Two patients required prolonged postoperative venoarterial (VA) ECMO support. Grade 3 primary graft dysfunction occurred in one patient; however, the clinical course improved following prolonged VA ECMO therapy. Five patients (83.3\%) required intervention for postoperative bleeding control; one of these patients died of uncontrolled bleeding concomitant with disseminated intravascular coagulation, on the 14th postoperative day, and we observed no other perioperative deaths. One patient died of carbapenem-resistant Acinetobacter baumannii bacteremia, a year postoperatively. The 1-month, 6-month, and 1-year survival rates were $83.3 \%, 83.3 \%$, and $66.7 \%$, respectively.

Conclusions: In view of the poor prognosis of IPAH, lung transplantation (1-year mortality rates $<40 \%$ ) merits consideration as a useful therapeutic option in this patient population. However, postoperative bleeding tends to occur in most patients; therefore, close monitoring is important during post-transplantation management.

Corresponding author: Sangbum Hong

E-mail: sbhong@amc.seoul.kr

(c) The Korean Society for Transplantation

This is an Open Access article distributed under the terms of the Creative Commons Attribution Non-Commercial License (http://creativecommons.org/licenses/by-nc/4.0/) which permits unrestricted non-commercial use, distribution, and reproduction in any medium, provided the original work is properly cited. 